\title{
Plasma Turbulence Imaging via Beam Emission Spectroscopy in the Core of the DIII-D Tokamak
}

\author{
George R. McKEE, Raymond J. FONCK, Deepak K. GUPTA, David J. SCHLOSSBERG, \\ Morgan W. SHAFER, Réjean L. BOIVIN ${ }^{1)}$ and Wayne SOLOMON ${ }^{2)}$ \\ University of Wisconsin-Madison, Madison, Wisconsin, 53706, USA \\ ${ }^{1)}$ General Atomics, San Diego, California, 92121, USA \\ 2) Princeton Plasma Physics Laboratory, Princeton, New Jersey, USA
}

(Received 2 December 2006 / Accepted 6 April 2007)

\begin{abstract}
Beam Emission Spectroscopy (BES), a high-sensitivity, good spatial resolution imaging diagnostic system, has been deployed and recently upgraded and expanded at the DIII-D tokamak to better understand density fluctuations arising from plasma turbulence. The currently deployed system images density fluctuations over an approximately $5 \times 7 \mathrm{~cm}$ region at the plasma mid-plane (radially scannable over $0.2<r / a \leq 1$ ) with a $5 \times 6$ (radial $\times$ poloidal) grid of rectangular detection channels, with one microsecond time resolution. BES observes collisionally-induced, Doppler-shifted $\mathrm{D}_{\alpha}$ fluorescence $(\lambda=652-655 \mathrm{~nm})$ of injected deuterium neutral beam atoms. The diagnostic wavenumber sensitivity is approximately $k_{\perp}<2.5 \mathrm{~cm}^{-1}$, allowing measurement of longwavelength $\left(k_{\perp} \rho_{I}<1\right)$ density fluctuations. The recent upgrade includes expanded fiber optics bundles, customdesigned high-transmission, sharp-edge interference filters, ultra fast collection optics, and enlarged photodiode detectors that together provide nearly an order of magnitude increase in sensitivity relative to an earlier generation BES system. The high sensitivity allows visualization of turbulence at normalized density fluctuation amplitudes of $\bar{n} / n<1 \%$, typical of fluctuation levels in the core region. The imaging array allows for sampling over 2-3 turbulent eddy scale lengths, which captures the essential dynamics of eddy evolution, interaction and shearing.
\end{abstract}

(c) 2007 The Japan Society of Plasma Science and Nuclear Fusion Research

Keywords: turbulence, plasma diagnostic, imaging, beam emission spectroscopy, density fluctuation, anomalous transport

DOI: $10.1585 /$ pfr.2.S1025

\section{Introduction}

Plasma turbulence plays a critical role in the confinement of energy, particles and momentum in magneticallyconfined fusion plasmas. Pressure gradients inherent to such plasmas are thought to drive drift-wave turbulence [1], which in turn drives cross-field transport at levels far exceeding the irreducible minimum of collisionally-driven "neoclassical" transport. Plasma turbulence is thought to be dominantly electrostatic in nature [2], and so radial transport arises from correlated fluctuations in the density, radial $\mathrm{E} \times \mathrm{B}$ velocity, temperature and parallel and poloidal velocities [3]. At higher normalized plasma pressure (beta), electromagnetic effects may also become important. Because of the central role that turbulent-driven transport plays in the confinement of fusion plasmas and therefore performance of fusion systems, it is necessary to thoroughly understand plasma turbulence from both a theoretical and experimental perspective. Because turbulence is manifest as fluctuations in the main plasma parameters of density, temperature, and velocity, it has been necessary and fruitful to deploy numerous fluctuation diagnostics on a variety of experiments to measure and characterize such turbulence so that a deeper understanding and,

author's e-mail: mckee@fusion.gat.com ultimately, a predictive capability can be developed. Such capability will require an aggressive program to validate nonlinear simulations of plasma turbulence and transport with detailed measurements of fluctuations, and their scaling properties, in well-characterized experiments.

Turbulence diagnostics have advanced significantly in capability and performance over the last couple of decades and are now allowing detailed measurements of fluctuation properties in high-performance fusion-grade plasmas over a wide range of wavenumbers, with good spatial localization for several fields (e.g., density and temperature).

Turbulence in magnetically-confined plasmas is a largely two-dimensional phenomenon, since the magnetic field defines an ignorable coordinate. Turbulent eddy structures have $k_{\|}<<k_{\perp}$, and so the most important turbulence dynamics take place in the plane perpendicular to the magnetic field. As such, it has been widely recognized that characterization of turbulence in the 2D radial-poloidal plane is of special importance and has motivated the development of 2D fluctuation diagnostics. Despite the significant development of fluctuation diagnostics, there are remarkably few that can measure fluctuations in 2D. Such diagnostics have included probe arrays [4], gas puff imaging systems [5-7] and an earlier generation beam emis- 
sion spectroscopy system [8-10]. These diagnostics were limited to the edge, separatrix, and scrape off regions or cold plasmas due to the various diagnostic limitations. 2D ECE Imaging systems are now providing electron temperature fluctuation measurements in the radial-poloidal plane in the core of the TEXTOR tokamak [11], though generally not with the time-resolution and sensitivity required for time resolved turbulence measurements.

The beam emission spectroscopy system at the DIII-D tokamak has been recently upgraded to substantially higher sensitivity. This dramatically enhanced signal-to-noise ratio of the upgraded system now allows for time-resolved imaging of turbulent density fluctuations into the core regions of low-confinement mode (L-mode) discharges. The first imaging measurements of core $(r / a<0.8)$ density fluctuations in a tokamak plasma were obtained recently [12]. This BES system has recently been expanded to nearly double the array size of this first phase. This is providing turbulence imaging measurements over a wider area, now covering a few turbulence correlation lengths in the radial and poloidal directions.

This paper presents and discusses these spatially expanded high-sensitivity 2D measurements of density fluctuations. Section 2 reviews the principles of the BES diagnostic and the main elements of the expanded, highsensitivity system. The characteristics of plasma turbulence in a set of typical low-confinement mode discharges is presented in Section 3, and imaging methods and measurements are presented in Section 4. The paper concludes with a discussion of applications of such measurements, future directions and summary in Section 5.

\section{Overview of the Beam Emission Spectroscopy Diagnostic}

Beam Emission Spectroscopy measures longwavelength $\left(k_{\perp} \rho_{I}<1\right)$ density fluctuations by observing collisionally-induced Doppler-shifted $\mathrm{D}_{\alpha}$ emission from injected neutral beam atoms $\left(n=3-2, \lambda_{o}=656.1 \mathrm{~nm}\right)$ [13]. The optical and detection systems allow for multi-channel measurements and good localization in the radial-poloidal plane. For the system discussed here, 32 channels are deployed in a 5 (radial) $\times 6$ (poloidal) $2 \mathrm{D}$ grid, plus two common-mode rejection channels [14]. Each channel images an approximately $0.9 \mathrm{~cm}$ (radial) $\times 1.2 \mathrm{~cm}$ (poloidal) region with channels located immediately adjacent to each other in each direction, for a total sampling area of approximately $5 \times 7 \mathrm{~cm}$. The spatial point spread function is radially dependent and is calculated using the optical sightline and neutral beam viewing geometry, magnetic field geometry, and optical ray tracing, and also includes finite excited state lifetimes [15], The actual sampled area for a given channel thus extends modestly beyond the directly imaged region. The $2 \mathrm{D}$ grid can be radially scanned on a shot-to-shot basis across the outboard midplane of the plasma (near $Z=0$ ) to provide measurements over

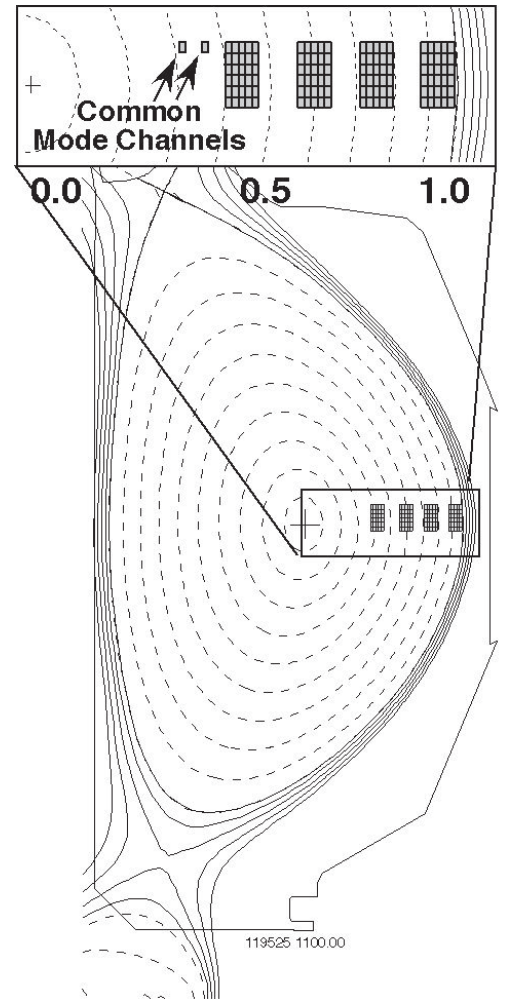

Fig. 1 Equilibrium reconstruction, and positions of $5 \times 6 \mathrm{BES}$ grid detection array for the four discharges presented.

the radial range $0.2<r / a<1$ (plasma minor radius, a), as well as into the scrape-off-layer region. The channel configuration for the discharges used for these imaging measurements is overlaid on a reconstruction of the equilibrium magnetic field shown in Fig. 1. Four repeat discharges were utilized with the radial position scanned from shot to shot between $r / a=0.4$ and $r / a=0.9$.

Details of the diagnostic system are discussed elsewhere $[16,17]$ and an overview is provided here. The observed emission results from an injected deuterium neutral beam with an energy of 70-80 keV $\left(v=2.6 \times 10^{6} \mathrm{~m} / \mathrm{s}\right)$, and $P_{\text {inj }} \approx 2.5 \mathrm{MW}$. Electron and ion collisions with beam atoms result in excitation and $\mathrm{D}_{\alpha}$ emission from the three beam-energy components. Emission is Dopplershifted over approximately $\lambda \approx 652-655 \mathrm{~nm}$. A highthroughput objective lens $(f=40 \mathrm{~cm}, f / 2)$ images light from the beam onto a set of fiber optic bundles with a magnification, $M \approx 3$. The sightline is approximately tangent to flux surfaces and is angled to match the dominant magnetic field pitch angle to provide good spatial resolution perpendicular to the field lines. The fibers are located on a motorized mount that can be remotely scanned between shots. Each detection channel consists of 11 1-mm plasticclad-silica fibers, $40 \mathrm{~m}$ in length, arranged in a 4:3:4 configuration, that convey the light to a remotely located spectroscopy lab.

Each detection channel consists of a collimating lens, 
interference filter, high-speed focusing lens and PIN photodiode. The interference filter is a custom-designed, high transmission filter that transmits light in the spectral range $\lambda=652-655.5 \mathrm{~nm}$, cutting off near $\mathrm{D}_{\alpha, 0}$. Specialized ultra low noise cryogenically-cooled transimpedance preamplifiers [18] convert the photodiode current to a voltage signal. Signal conditioning electronics then frequency filter and further amplify the signal. The signal is digitized using multichannel simultaneous digitizers (D-tAcq Solution Inc.) Two 14-bit, 16-channel digitizer boards (ACQ16PCI) utilize synchronized external clocks and triggers to insure that all 32 channels are sampled at $1 \mathrm{MHz}$ on a common time-base, crucial for cross correlation and cross phase measurements. Each board has $128 \mathrm{MB}$ of on-board memory, allowing for $64 \mathrm{MS}$ for up to $4.2 \mathrm{sec}$ of synchronized data acquisition at $1 \mathrm{MHz}$, the typical sampling rate.

The newly implemented BES system has been substantially upgraded from an earlier generation system at DIII-D [10, 16], and relocated to a larger laboratory to facilitate further expansion. The upgraded system provides substantially increased sensitivity to small-amplitude density fluctuations. The primary elements of the upgrade are higher throughput fibers (11 1-mm fibers/channel, compared to 4), higher transmission, wider-band interference filters, ultra high-speed collection lenses and a larger area detector. The net result of these various improvements was an approximately 5-10 increase in the measured signal level, and an associated increase of 30-50 in fluctuation power, a consequence of the relationship between electronic noise, photon noise and density fluctuation signal. See Ref. 12 for a detailed discussion of the noise components and SNR improvements. The first phase of the upgraded BES system consisted of 16 channels arranged in a $4 \times 4$ grid, while the recently expanded system consists of 32 high-sensitivity channels $(5 \times 6$ grid plus two commonmode channels).

Two common-mode rejection channels have been deployed with the recently expanded system. These channels are located approximately five and $10 \mathrm{~cm}$ inboard of the main $5 \times 6$ channel grid, respectively, and are shown in the configuration in Fig. 1. The purpose of these channels is to measure and isolate any fluctuation components on the neutral beam itself that do not represent local plasma fluctuations and thus should be subtracted from the measured signals [14]. Such common-mode fluctuations arise predominantly from fluctuations in the neutral beam ion source [19], as well as large-amplitude edge fluctuations that become imprinted on the beam due to fluctuating beam attenuation. Such fluctuations are superimposed on the measured local fluctuations and can thus complicate analysis. The common-mode channels allow for the identification of spectral signals that are common to all channels. They are separated radially from the main $5 \times 6$ array by a distance that is larger than the radial correlation length of turbulent eddies. Therefore, any signal common between these channels and the array can be rejected as common-

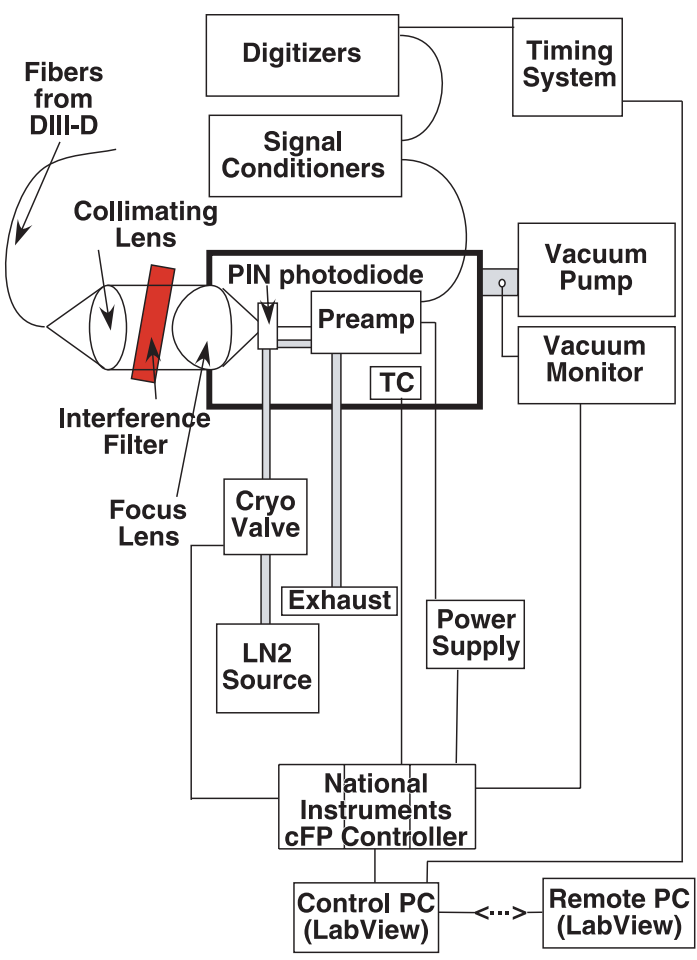

Fig. 2 Block diagram of the main components of the Beam Emission Spectroscopy detection and control system, including spectrometers, detectors, electronics, temperature regulators, timing, and control components.

mode signal. The channels need to be accurately calibrated to apply this procedure. An example of this is presented in Section 3.

An all-new control system has been implemented to control timing, detector temperature control and AC power to various system components, and provide for safety of the equipment. This system incorporates several National Instruments compact Field Point (cFP) components (relay, thermocouple, analog input modules) and a timing board, that have been integrated with the LabView virtual instrument software package for interface and control running on a host PC. The control system allows for fully remote operation of the BES system, system protection, and recording of diagnostic system parameters. The system controls LN2 flow to the detectors and preamplifiers to maintain a temperature near $140 \mathrm{~K}$ to minimize e-noise and photodiode dark current (all cooled components are located in a vacuum box to prevent condensation). Power is cycled to cryogenic solenoid valves in response to real-time temperature measurements. The timing system allows for data acquisition to commence at any point during the plasma discharge with a precision of 1 microsec. A block diagram illustrating the detection and control system is shown in Fig. 2.

\section{Measured Turbulence Characteris- tics}

The first core turbulence imaging measurements with the upgraded and expanded $5 \times 6$ channel BES array were 
obtained in a set of low-confinement (L-mode) discharges on DIII-D. The primary goal of the experiment was to determine the toroidal Mach number dependence of turbulence and transport; the toroidal Mach number was varied by adjusting the torque applied to the plasma using the newly implemented co-current and counter-current neutral beam injection capability on DIII-D [20]. The array was scanned shot-by-shot over four radial locations, as indicated in Fig. 1. The discharge parameters after current flattop were: $I_{\mathrm{p}}=1.0 \mathrm{MA}, B_{\mathrm{T}}=-2.0 \mathrm{~T}, P_{\text {inj }}=5.0 \mathrm{MW}$, $n_{\mathrm{e}, \mathrm{o}}=2.9 \times 10^{19} \mathrm{~m}^{-3}, T_{\mathrm{e}, \mathrm{o}} \approx 2.2 \mathrm{keV}, T_{\mathrm{i}, \mathrm{o}} \approx 2.7 \mathrm{keV}$.

A spectrogram of the temporally and spectrally resolved density fluctuations is shown in Fig. 3. These fluctuations, obtained near $r / a=0.65$, are displayed over the frequency range $15-450 \mathrm{kHz}$, from $0.32-3.3 \mathrm{sec}$. The density fluctuations associated with turbulence are clearly evident as a broadband feature that increases in frequency as the discharge evolves. The central frequency increases from less than $100 \mathrm{kHz}$ in the early phase $(t<1.0 \mathrm{sec})$ to over $200 \mathrm{kHz}(t>1.5 \mathrm{sec})$ primarily as the $\mathrm{E} \times \mathrm{B}$ poloidal velocity and resulting Doppler shift increase with increasing plasma rotation. The frequency range extends from several tens of $\mathrm{kHz}$ up to roughly $400 \mathrm{kHz}$, indicating a highly nonlinear saturated turbulence state. Also evident is a sharp reduction in the fluctuations near $2.55 \mathrm{sec}$ when the plasma undergoes a transition from a low-confinement mode (L-mode) to a high-confinement mode (H-mode). While the LH transition is primarily associated with the rapid development of a strong radial electric field shear layer and fluctuation suppression near the plasma pedestal $(r / a>0.9)$ [21], it is clear that fluctuations in this deeper core region are also affected and suppressed on a short time scale.

Oscillations arising in the neutral beam source itself are also observed in the frequency range $0-70 \mathrm{kHz}$

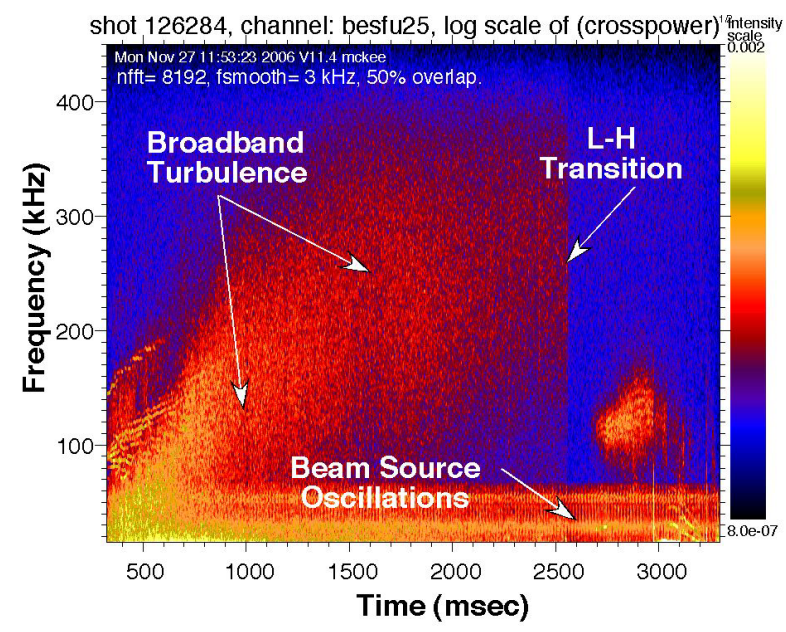

Fig. 3 Spectrogram of measured density fluctuations at $r / a=$ 0.65 showing evolution of the turbulent density fluctuations. continuously throughout the discharge. The frequencyintegrated amplitude of the beam oscillations is typically $\bar{n}_{\text {beam }} / n_{\mathrm{o}}<1 \%$, but relative to local turbulent fluctuations, these non-local beam oscillations can be significant in the core plasma. In cases like that shown in Fig. 3, the beam oscillations can be spectrally isolated from the higher-frequency turbulent fluctuations, but in lower rotation plasmas, it becomes necessary to measure and subtract off the beam-source oscillation component using the common-mode rejection channels discussed previously. Fig. 4 demonstrates the common-mode isolation and subtraction procedure. Fig. 4(a) shows two ensemble-averaged cross-power spectra: one from two poloidally-separated channels $(\triangle Z=1.2 \mathrm{~cm}$, data from same channels as shown in Fig. 3), and one between one of those channels and one of the common-mode channels $(\Delta R=10 \mathrm{~cm}, \Delta Z=0 \mathrm{~cm})$. The local cross-power spectrum (red-solid) exhibits both a broadband turbulence component $(80-400 \mathrm{kHz}$, dominantly) along with the beam oscillation component, or common-mode spectrum $(f<70 \mathrm{kHz})$ ). The commonmode spectrum (blue-dashed) exhibits only the common-
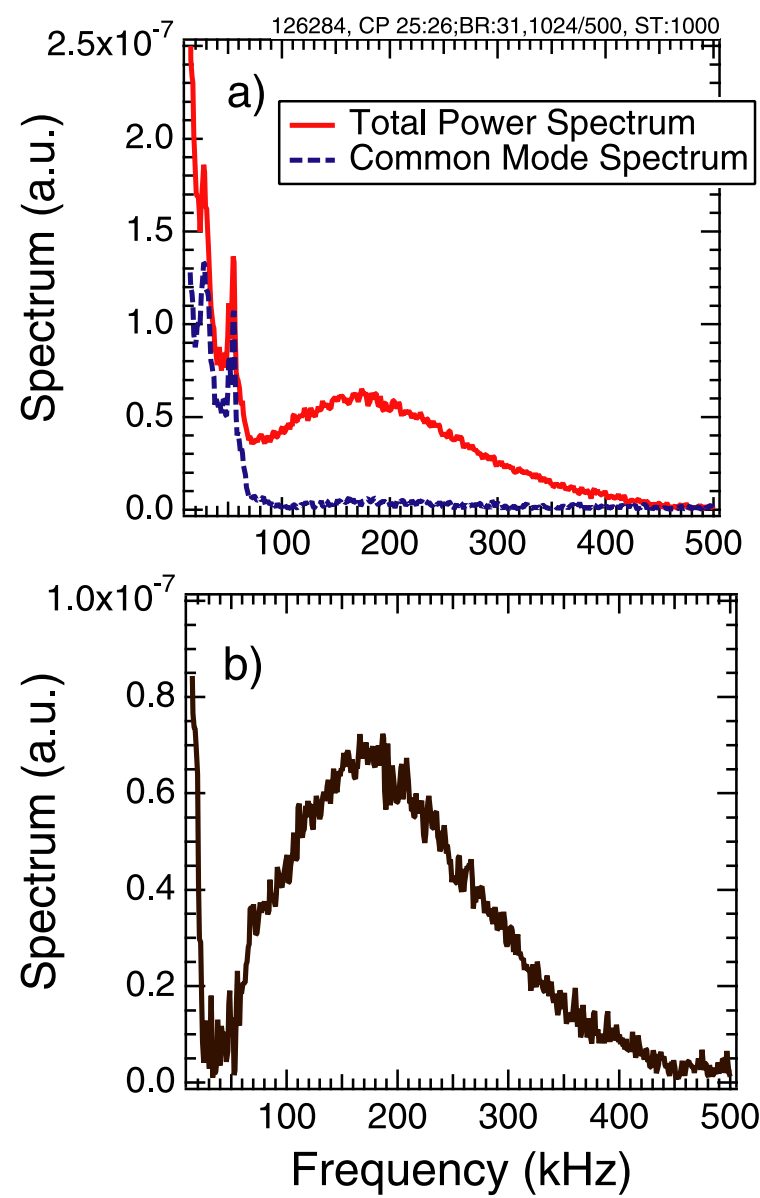

Fig. 4 (a) Cross-power fluctuation spectrum from adjacent channels (red) and two widely spaced channels (blue) showing the common-mode component; (b) Local fluctuation spectrum after subtraction of the common mode component. 

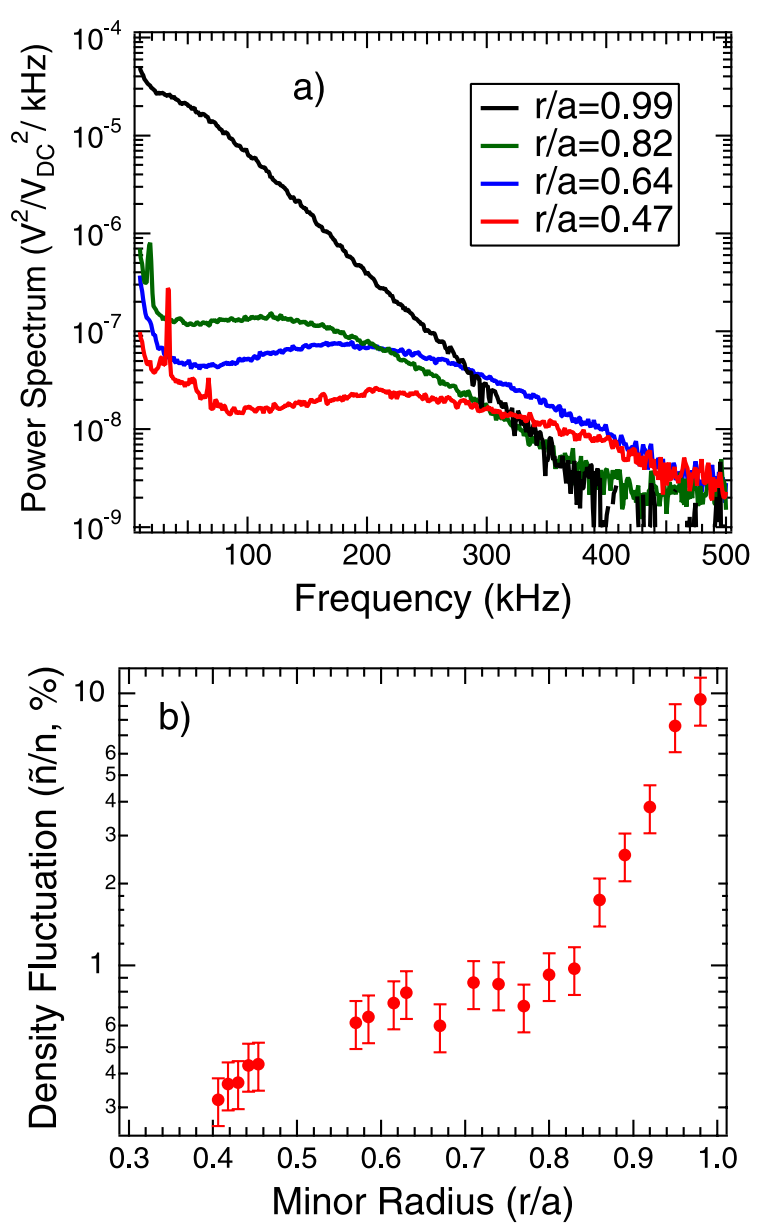

Fig. 5 (a) Density fluctuation power spectra at four radial locations; (b) Spectrally integrated fluctuation amplitude profile $(t=1.0-1.5 \mathrm{sec})$.

mode beam oscillations because the two channels are separated by a distance large compared to the radial correlation length of the turbulence. Fig. 4(b) shows the local fluctuation spectrum after the common-mode (dominantly beam oscillation) component is subtracted off. It can be seen that the broadband turbulence spectrum extends down into the lower frequency range $(f<70 \mathrm{kHz})$ where the beam oscillations dominate, though the resulting spectrum is of relatively low-amplitude in this case. The resulting spectrum is relatively noisy at this lower frequency since two larger signals are being subtracted to obtain the residual local fluctuation component.

The power spectra of the broadband turbulence vary strongly with radius. Figure 5 displays the power spectra at four radii (integrated over $t=1000-1500 \mathrm{~ms}$ ) extending from mid-radius to near the plasma edge. It can be readily observed that the fluctuation amplitude increases strongly towards the plasma edge, and that the spectrum is Doppler-shifted towards higher frequency towards the mid-radii of the plasma (the common-mode beam oscillations have been subtracted off using the procedure discussed above, where significant). The integrated fluctua-
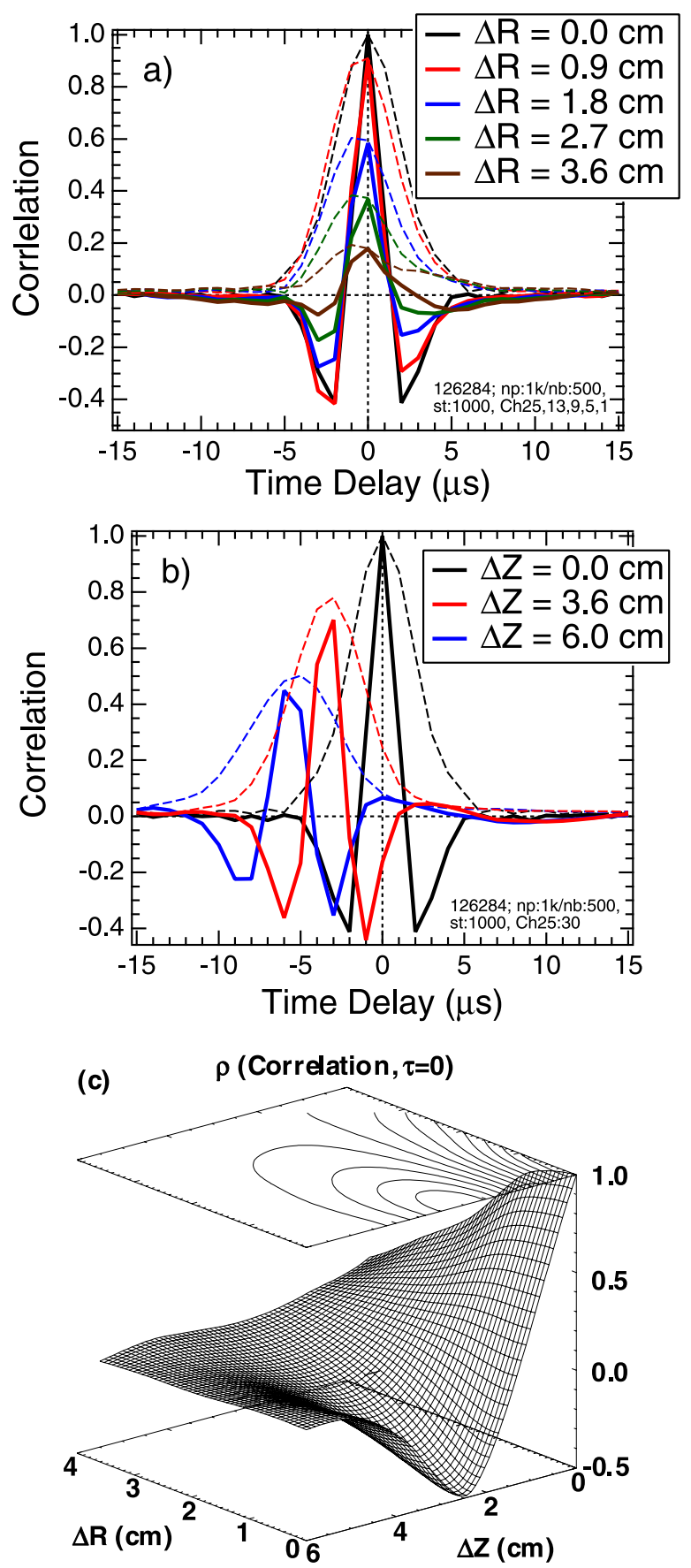

Fig. 6 (a) Radial time-lag cross-correlation functions $(r / a=0.6)$; solid lines are cross-correlation functions, dashed lines envelope functions; (b) Poloidal cross-correlation functions; (c) 2D (radial-poloidal) spatial cross-correlation function at time lag, $\tau=0$.

tion amplitude is likewise shown in Fig. 5(b), showing a factor of 30 variation from $r / a=0.4$ out to the edge, which is typical of L-mode plasma conditions [22, 23].

The radial and poloidal time-lag cross-correlation functions exhibit a strong asymmetry due primarily to the large poloidal $\mathrm{E} \times \mathrm{B}$ advection of turbulent structures. The radial cross correlation functions at increasing radial separation near $r / a=0.6$ are shown in Fig. 6(a), and the poloidal cross correlation functions in Fig. 6(b). The solid 
lines represent the cross-correlation functions, and the associated dashed lines represent the respective envelope functions (defined using the Hilbert transform). The radial cross-correlation functions all peak at a time lag of zero, reflecting zero time-averaged radial propagation of turbulent eddy structures. The radial correlation length, defined as the $1 / \mathrm{e}$ decay distance, is $L_{\mathrm{c}, \mathrm{r}} \approx 2.5 \mathrm{~cm}$ here. In contrast, the poloidal cross-correlation functions peak at monotonically decreasing time-lags as a result of the strong poloidal $\mathrm{E} \times \mathrm{B}$ advection. The corresponding poloidal correlation length, taken again as the 1/e decay distance at zero time lag, is $L_{\mathrm{c}, \theta} \approx 3.0 \mathrm{~cm}$. The decorrelation time of the turbulence is defined as the time-lag at which the cross-correlation has decayed to $1 /$ e. As a result of the strong poloidal advection, the poloidal cross-correlation amplitudes do not decay to $1 / \mathrm{e}$ over the $6 \mathrm{~cm}$ observation range, but rather decay to only 0.5 . The decorrelation time is estimated by extrapolation to be about $\tau_{\mathrm{c}} \approx 8 \mu \mathrm{s}$. The $\tau=02 \mathrm{D}$ spatial cross-correlation function is shown in Fig. 6(c), showing the expected wavelike structure in the poloidal direction, with a monotonically decaying radial structure. This graph was constructed by computing the time-lag cross correlation function between a reference channel at the corner of the 2D array and all other channels, evaluating at $\tau=0$, and smoothing the resulting $2 \mathrm{D}(5 \times 6)$ graph using a minimum curvature spline surface routine.

The poloidal advection velocity of the turbulent eddies, or group velocity, can be straightforwardly determined from the inverse of the slope of the peak time-lag vs. poloidal separation. The measured group velocity profile is shown in Fig. 7. In these co-injection plasmas, the poloidal velocity peaks near $r / a=0.45$ at roughly $14 \mathrm{~km} / \mathrm{s}$, and decays to about $5 \mathrm{~km} / \mathrm{s}$ near the edge and also shows a small by significant drop towards the center, inboard from the peak at $r / a=0.45$. The poloidal velocity of turbulence is governed primarily by the radial electric field, which naturally drops to zero at the magnetic axis. The

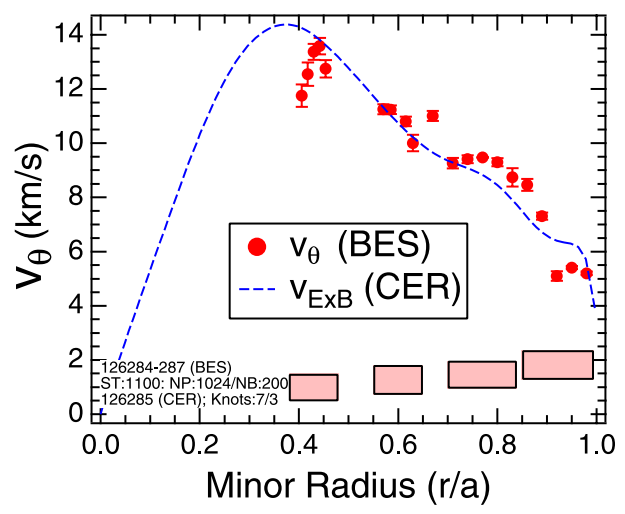

Fig. 7 Poloidal turbulence velocity profile, and $\mathrm{E} \times \mathrm{B}$ velocity measured from charge exchange recombination spectroscopy. Horizontal bars at bottom represent the radial extent of BES array for the four shots examined.
$\mathrm{v}_{\mathrm{E} \times \mathrm{B}}$ profile is simultaneously measured using charge exchange recombination spectroscopy [24] and is overlaid in Fig. 7 as a dashed line. The radial electric field is measured from the carbon ion force balance equation using the directly measured toroidal and poloidal rotation velocities and pressure gradient. The $\mathrm{E} \times \mathrm{B}$ velocity is then simply calculated as $v_{E \times B, \theta}=\frac{\vec{E}_{r} \times \vec{B}_{T}}{B^{2}}$. The very good quantitative agreement between these two independent measurements demonstrates that the turbulence advects poloidally at the $\mathrm{E} \times \mathrm{B}$ velocity. The fluctuations are propagating in the laboratory frame in the ion diamagnetic direction, which is the same direction as the $\mathrm{E} \times \mathrm{B}$ velocity driven by the coinjecting neutral beams on DIII-D. The turbulence $S(k, \omega)$ spectrum exhibits a wide range of wavenumbers and frequencies, as seen in the power spectra in Fig. 5 and spatial cross-correlation in Fig. 6(c). At a given location, all wavenumbers/frequencies are advected nearly uniformly at the derived velocity shown. In other words, there is little dispersion over much of the core region of the plasma. This is demonstrated by a monotonically increasing linear phase shift in the cross power spectra measured from poloidally-separated channels. This is to be expected since any dispersive effects are predicted to be on the scale of the diamagnetic velocity for drift wave turbulence, thought to dominate the broadband fluctuations; this is typically far smaller than the $\mathrm{E} \times \mathrm{B}$ velocity. An exception may occur near the plasma edge region where the $\mathrm{E} \times \mathrm{B}$ velocity becomes smaller, while diamagnetic drifts become larger and dispersive effects have been observed [25].

\section{Turbulence Images}

The general characteristics of the turbulence were presented in Section 3 and illustrated the ensemble-average properties of the broadband fluctuations associated with plasma turbulence. Correlation lengths are observed to be a few $\mathrm{cm}$, decorrelation times near $10 \mu \mathrm{s}$, and poloidal advection speeds of $5-10 \mathrm{~km} / \mathrm{s}$ for these L-mode discharges on DIII-D. The time-resolved dynamics of the turbulence are necessary to identify and quantify several important nonlinear characteristics of the turbulence. These include, for example, zonal flows and geodesic acoustic modes, energy cascades, and may facilitate direct turbulent particle flux measurements. To begin to address these issues, and to illustrate the time-resolved eddy interactions such as shearing and accretion, images and movies of the turbulence in the core plasma region have been developed from the recently upgraded and expanded BES density fluctuation measurements. Figure 8 displays the frequencyfiltered time-series fluctuation signal over $150 \mu$ s from three poloidally-separated channels near $r / a=0.8$. The signal-to-noise ratio for this time-resolved data is approximately 2 across the frequency range considered (60$300 \mathrm{kHz}$, where the plasma fluctuation signal dominates), with noise composed primarily of photon noise and a small electronic noise component. Thus, the signal appears tur- 


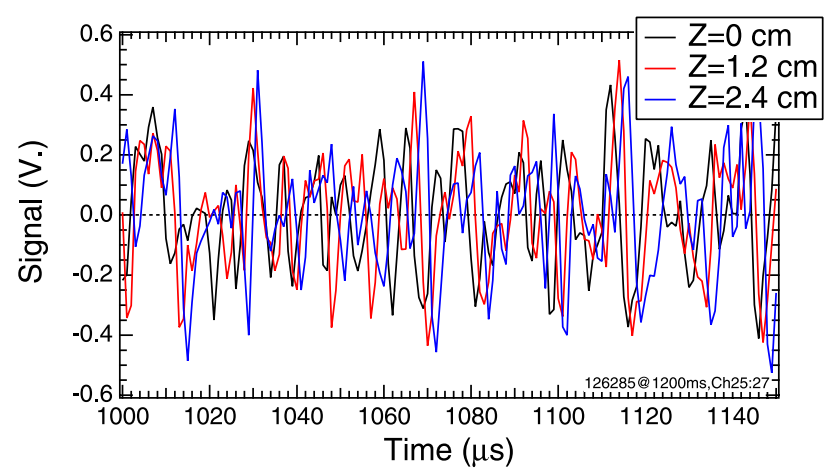

Fig. 8 Time-resolved fluctuation measurements at three poloidally-displaced channels near $r / a=0.82$.

bulent, as such, and is dominated by the local plasma density fluctuations. The high coherency of the signals is readily apparent, with structures strongly correlated from one channel to the next. The poloidal advection is likewise apparent as a time-shift of the coherent structures between each channel.

Images of the turbulence are constructed from the spatially resolved fluctuation measurements as has been discussed previously $[9,10,12]$, Each signal is first frequencyfiltered over the relevant spectral range. This is identified by determining where the broadband turbulence dominates the spectrum, as measured via the respective coherency spectra between adjacent channels. The RMS values of signals from the 30 channels in the $5 \times 6$ grid are then normalized to eliminate channel-to-channel variations. A 2D spline fit is performed with the discrete $5 \times 6$ channel grid using a minimum curvature spline surface routine (MIN_CURVE_SURF routine in IDL) to generate a 50×60 point interpolated grid. This is accomplished by constructing a set of basis functions with corresponding coefficients, solving for the coefficients, and evaluating the resulting surface at the desired points. The resulting array is then linearly interpolated in $2 \mathrm{D}$ to expand to the desired pixel size.

A selected set of turbulence images generated with the above method is shown in Fig. 9. Each image covers an approximately $4.5 \mathrm{~cm} \times 7.2 \mathrm{~cm}$ (radial $\times$ poloidal) region along the outboard midplane, with locations shown on the equilibrium reconstruction in Fig. 1. The aspect ratio is nearly preserved in these images. Four very similar (repeat) discharges were used to obtain this sequence, with the radial position of the fiber array moved shot-to-shot to the respective locations. Images in each vertical column were obtained from one discharge and each frame is separated by $2 \mu \mathrm{s}$, with time progressing downwards. Here, the green color represents an equilibrium density value, while red represents positive density fluctuations, and blue negative. The eddy structures are generally propagating in a vertically upward direction $(\mathrm{E} \times \mathrm{B}$ direction) in each frame. The individual turbulent eddies are most easily identified
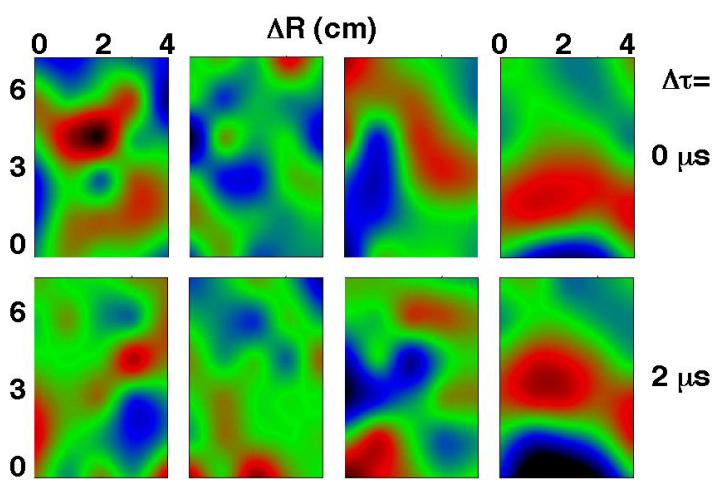

$2 \mu s$
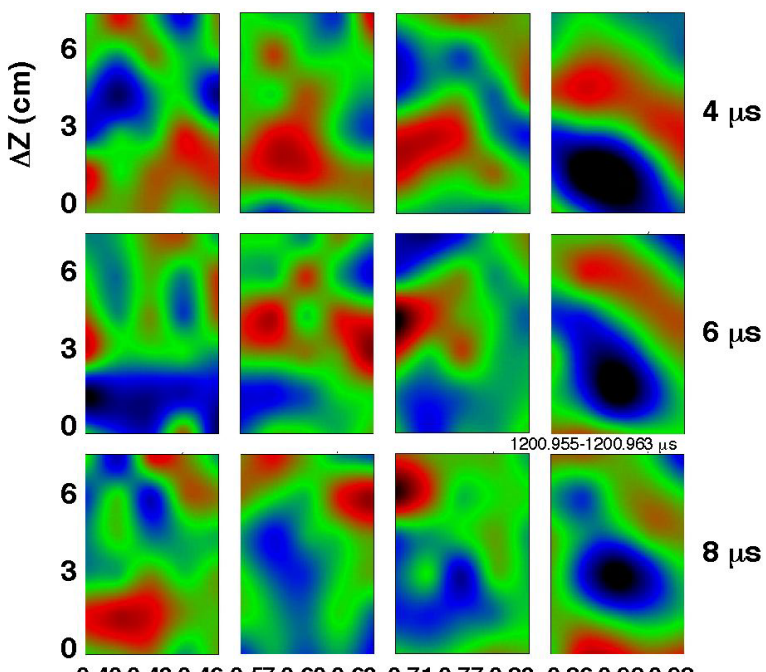
Minor Radius (r/a)

Freq.
Range

$\begin{array}{lllllll}0.400 .43 & 0.46 & 0.57 & 0.600 .63 & 0.710 .770 .83 & 0.86 & 0.920 .98\end{array}$ $\begin{array}{llll}70-375 \mathrm{kHz} & 70-425 \mathrm{kHz} & 60-400 \mathrm{kHz} & 10-300 \mathrm{kHz}\end{array}$

Fig. 9 Images of turbulent density fluctuations from four similar discharges at four radial locations, each frame separated by 2 microsec (color-coding: green-neutral, red-positive, blue-negative). Frequency filter range for each set of images is shown below figures.

in the radially outermost set of data, where the poloidal flow shear can be seen to be tearing the eddies apart. The eddy structures at the inner radii undergo significant deformation from frame-to-frame, reflecting the complex interactions of the eddies. The structure scale lengths and lifetimes show general consistency with the ensemble averaged measurements presented earlier, but here the strong temporal dynamics and interactions can be directly observed. Time-resolved visualizations have been made from these images and can be obtained [26].

\section{Discussion and Conclusion}

2D turbulence imaging data allows for far more detailed study of turbulence than is possible with zero or 1D measurements. The Beam Emission Spectroscopy density fluctuation diagnostic system at DIII-D has recently been upgraded to achieve significantly higher sensitivity to small-scale fluctuations, and has been expanded to 32 high-sensitivity channels. The channels are deployed in a $5 \times 6$ channel grid covering an approximately $4.5 \times 7.2 \mathrm{~cm}$ 
region along the outboard midplane of the tokamak that can be radially scanned from shot-to-shot. Two channels are deployed at radially inboard locations to monitor and isolate common-mode fluctuations from the neutral beam source or edge turbulence imprinted on the beam. The enhanced sensitivity now allows for time-resolved measurements of density fluctuations at normalized fluctuation amplitudes $\bar{n} / n \leq 1 \%$. The $2 \mathrm{D}$ measurement capability in the radial-poloidal plane allows for detailed characterization of the inherently 2D turbulence in magnetically-confined plasmas. This allows for direct measurement of such quantities as the equilibrium flow shear of the turbulence, 2D correlation functions and $S\left(k_{r}, k_{\theta}\right)$ wavenumber spectra, zonal flows and geodesic acoustic modes and nonlinear energy transfer.

Newly developed routines are being applied to measure the time-resolved 2D velocity field of the turbulent eddy structures $[27,28]$. The inferred velocity field may allow for direct measurements of turbulent-driven particle flux, zonal flow measurement, and can provide an additional field describing the turbulence for use with advanced nonlinear analysis routines. Future studies will concentrate on the 2D fluctuation dynamics leading up to and across the LH transition, as well as development of the H-mode pedestal, zonal flow properties, turbulence dynamics during the formation of internal transport barriers, and dimensionless scaling properties of turbulence and transport. Together, these studies will play a crucial role in the validation of sophisticated nonlinear turbulence simulations and should aid the development of a predictive capability for turbulent-driven transport in fusion plasmas.

\section{Acknowledgments}

The authors would like to thank the DIII-D program for its technical and engineering support for this diagnostic development program as well as the scientific support for experimental run time for these and related research activities. This activity supported by U.S. DOE under DEFG02-89ER53296 and DE-FC02-04ER54698.

[1] W. Horton, Rev. Mod. Phys. 71, 735 (1999).

[2] C. Petty, Phys. Plasmas 11, 2514 (2004).

[3] B.A. Carreras, IEEE Trans. Plasma Sci. 25, 1281 (1997).

[4] S.J. Zweben and R.W. Gould, Nucl. Fusion 25, 171 (1985).
[5] S.J. Zweben et al., Phys. Plasmas 9, 1981 (2002).

[6] R.J. Maqueda, G.A. Wurden, D.P. Stotler, S.J. Zweben et al., Rev. Sci. Instrum. 74, 2020 (2003).

[7] J.L. Terry, S.J. Zweben, K. Hallatschek et al., Phys. Plasmas 10, 1739 (2003).

[8] C. Fenzi, R.J. Fonck, M. Jakubowski and G.R. McKee, Rev. Sci. Instrum. 72, 988 (2001).

[9] G.R. McKee, C. Fenzi, R.J. Fonck, IEEE Trans. Plasma Sci. 30, 62 (2002).

[10] G.R. McKee, C. Fenzi, R.J. Fonck, M. Jakubowski, Rev. Sci. Instrum. 74, 2014 (2003).

[11] H. Park, E. Mazzucato, T. Munsat et al., Rev. Sci. Instrum. 75, 3787 (2004).

[12] G.R. McKee, R.J. Fonck, D.K. Gupta et al., Rev. Sci. Instrum. 77, 10F104 (2006).

[13] R.J. Fonck, P.A. Dupperex, S.F. Paul, Rev. Sci. Instrum. 61, 3487 (1990).

[14] R.D. Durst. R.J. Fonck, G. Cosby, H. Evensen, Rev. Sci. Instrum. 63, 4907 (1992).

[15] M.W. Shafer, R.J. Fonck, G.R. McKee, D.J. Schlossberg, Rev. Sci. Instrum. 77, 10F110 (2006).

[16] G. McKee, R. Ashley, R. Durst, R. Fonck et al., Rev. Sci. Instrum. 70, 913 (1999); G. McKee, R. Ashley, R. Durst, R. Fonck et al., Rev. Sci. Instrum. 70, 2179 (1999).

[17] D.K. Gupta, R.J. Fonck, G.R. McKee et al., Rev. Sci. Instrum. 75, 3493 (2004).

[18] R.J. Fonck, R. Ashley, R. Durst, S.F. Paul and G. Renda, Rev. Sci. Instrum. 63, 4924 (1992).

[19] R. Hong and H.K. Chiu, "Effects of Operating Parameters on the Beam Species of DIII-D Neutral Beam Ion Sources" Proc. of 19th IEEE/NPSS Symp. on Fusion Engineering, Atlantic City, New Jersey, 2002

[20] T.C. Luce, K.H. Burrell, R.J. Buttery et al., Proc. IAEA Fusion Energy Conf. "Dependence of Confinement and Stability on Variation on the External Torque in the DIII-D Tokamak," PD-3 (2006).

[21] R.A. Moyer K.H. Burrell, T.N. Carlstrom et al., Phys. Plasmas 2, 2397 (1995).

[22] R.J. Fonck, G. Cosby, R.D. Durst et al., Phys. Rev. Lett. 70, 3736 (1993).

[23] G.R. McKee, C.C. Petty, R.E. Waltz et al., Nucl. Fusion 41, 1235 (2001).

[24] K.H. Burrell, Phys. Plasmas 6, 4418 (1999).

[25] C. Fenzi, G.R. McKee, R.J. Fonck et al., Phys. Plasmas 12, 062307 (2005).

[26] Available online from journal, or http://fusion.gat.com/diiid/BESMovies

[27] G.R. McKee, R.J. Fonck, D.K. Gupta et al., Rev. Sci. Instrum. 75, 3490 (2004).

[28] T. Munsat and S.J. Zweben, Rev. Sci. Instrum. 77, 103501 (2006). 